\title{
WHAT IS NEW ABOUT THE NEW COMET YANAKA (1988r)?
}

\author{
J. Mayo Greenberg \\ Laboratory Astrophysics, Huygens Laboratorium, Leiden University, P.O. Box 9504, 230 RA Leiden, The Netherlands \\ AND \\ P. D. Singh AND A. A. DE AlmeIDA \\ Institute of Astronomy and Geophysics, University of Såo Paulo, Caixa Postal 9638, CEP 01065 Såo Paulo, Brazil \\ Received 1993 April 22; accepted 1993 June 11
}

\begin{abstract}
A deficiency in the observed abundances of $\mathrm{C}_{2}$ and $\mathrm{CN}$ in comet Yanaka (1988r) is explained in terms of the properties of its organic refractory dust component and the fact that it is a new comet. Because the major fraction of the carbon in comets is in a mixture of complex organic molecules which earlier explained the "missing" carbon mystery it is shown that substantial carbon depletions are impossible. The low $\mathrm{C}_{2}$ and $\mathrm{CN}$ production rates may be understood as consequences of Yanaka (1988r) being a new comet in combination with the small central area of coma observed. The surface of new comets resulting from cosmic-ray processing in the Oort cloud gives rise to dust which is relatively nonfragile and which remains relatively large and cool within the limits of a small diaphragm making the comet appear to be dust poor. This dust yields smaller abundances of carbon-rich species by evaporation than customary for the smaller and hotter fragmented particles further out in the coma.
\end{abstract}

Subject headings: comets: individual (Yanaka 1988r) - dust, extinction - ISM: molecules

\section{INTRODUCTION}

The deficiency of $\mathrm{C}_{2}$ and $\mathrm{CN}$ observed by Fink (1992) in Comet Yanaka (1988r) was interpreted very provocatively as implying that it was one of "a new class of carbon poor comets." The first question to be asked is whether $\mathrm{C}_{2}$ and $\mathrm{CN}$ are proper probes of the total carbon abundance, and the second question is, if they are not, what is the real significance of their relative underabundance in this comet?

Since comets are presumed to have formed out of interstellar material in the protosolar nebula, and since there is no interstellar cloud at the present time which shows a significant depletion in carbon-if one includes what is in the dust-it seems very unlikely that there can exist a comet which is truly deficient in carbon. We must therefore search for a more reasonable way to explain how comet Yanaka (1988r) can be deficient in $\mathrm{C}_{2}$ and $\mathrm{CN}$ by one or two orders of magnitude and yet be a normal comet insofar as its total carbon content is concerned.

\section{WHERE IS MOST OF THE CARBON IN COMETS?}

In interstellar clouds a major fraction of the carbonprobably $60 \%$ or more of the total cosmic abundance--is bound up in the dust in some form of carbonaceous material (Greenberg 1982a, 1991; Mathis, Rumpl, \& Nordsieck 1977; Chlewicki 1986). So it is not in the gas molecule abundances (van Dishoeck et al. 1993) that we should be looking for such large deviations in carbon as attributed to comet Yanaka (1988r) but rather in some property, intrinsic or observational, of the dust. It will turn out, in fact, that the explanation proposed here for the $\mathrm{C}_{2}, \mathrm{CN}$ deficiencies will automatically imply that the $\mathrm{C}_{3}$ would also have been deficient whether or not the comet was faint or the spectrum lay outside the available spectral region. We note that the "carbon depletion mystery" posed by the anomalously low (compared with solar system abundances) $\mathrm{C}: \mathrm{O}$ ratio derived from the observed "volatile" carbon-bearing species (not only $\mathrm{CO}$ but also including $\mathrm{C}, \mathrm{C}^{+}$, etc.) was automatically explained by the prediction of the interstellar dust model of comets wherein the major carbonbearing species were the organic refractory interstellar grain mantles (Greenberg 1983). This has subsequently been substantiated by in situ measurements (Kissel \& Krueger 1987; Jessberger, Kissel, \& Rahe 1989; Geiss 1987).

The fact that $\mathrm{H}_{2} \mathrm{O}$ is the most abundant volatile species in comets supports the idea that comets are aggregates of interstellar dust because, while $\mathrm{H}_{2} \mathrm{O}$ as an interstellar gas phase molecule is nowhere as abundant as $\mathrm{CO}$, it is the most abundant interstellar dust mantle molecule where, in fact, it is made (Greenberg 1982a; d'Hendecourt, Allamandola, Greenberg 1985). As summarized in Mumma et al. (1993), $\mathrm{H}_{2} \mathrm{O}$ is just one of the many molecules which appear to have preserved their interstellar proportions during and after the comet was formed. For example, Larson et al. (1989) find similar $\mathrm{CH}_{4}$ abundances in Halley (a periodic comet) and in the dynamically new comet Wilson (19861), and both are inconsistently too low compared with the higher values predicted by equilibrium models and much too high for the lower disequilibrium condensation values in the solar nebula.

The similarity between the $3.4 \mu \mathrm{m}$ emission in old (periodic) Halley and in new Wilson (19861) and the $3.4 \mu \mathrm{m}$, absorption by interstellar dust are further confirmation that the organic refractories were incorporated along with the volatile ices, as outer dust mantles, at the time of formation. As far as carbon itself is concerned, Fink's (1992) suggestion of a possible chemical inhomogeneity in the early solar system where comets were formed is contradicted by Uranus and Neptune being pretty much the same (Pollack \& Bodenheimer 1989).

\section{ORGANIC REFRACTORY DUST SOURCES OF $\mathrm{C}_{2}$, CN}

As a consequence of the above arguments we look for an explanation of the "depletion factor" for $\mathrm{C}_{2}$ and $\mathrm{CN}$ in some 
aspect of the dust. In comet $\mathrm{P} / \mathrm{Halley}$ it was noted that $\mathrm{C}_{2}$ and $\mathrm{CN}$ as well as $\mathrm{C}_{3}$ and even $\mathrm{CO}$ were wholly or partially distributed molecules following the dust outward flow. They therefore have been considered to have been produced by evaporation and pyrolysis and subsequent photolysis of some of the less refractory organic compounds in the dust (Greenberg 1983; Greenberg \& Hage 1990; Briggs et al. 1992; Clairemidi, Rouffelot, \& Moreels 1991; Eberhardt et al. 1987). A'Hearn and coworkers (A'Hearn et al. 1986a, b) and Singh, de Almeida, \& Huebner (1993) support the idea that $\mathrm{CN}$ and $\mathrm{C}_{2}$ jets in Halley come from dust. Since comet Yanaka (1988r) is a new comet (Komet Tsirk 1988), perhaps its outer layers have undergone sufficient modification by cosmic radiation during its $4.5 \mathrm{Gyr}$ in space to strongly change its properties relative to the unexposed interior. It may be inferred from the work of Strazzulla \& Johnson (1991) that the cosmic rays "carbonize" the already existing organic component of the interstellar dust in the comet, that is, deplete the elements $\mathrm{H}, \mathrm{N}$, and $\mathrm{O}$ (relative to $\mathrm{C}$ ). This leads to much more refractory molecules with less capability of evaporation and breakup into smaller molecular groups like $\mathrm{C}_{2}$. At the same time, however, that the cosmic rays are carbonizing the original (interstellar) organics they are producing within the ices a new population of "fresh" organic refractory molecules which probably resemble the precarbonized organics. This results in a replacement or substitution of the old $\mathrm{C}_{2}, \mathrm{CN}$-producing organics by fresh ones. We conclude that the net result of the cosmic rays may not in itself alter the efficiency of $\mathrm{C}_{2}, \mathrm{CN}$ production by as much as the orders of magnitude required. This is confirmed by the fact that no such deficiency is observed in another new comet, Yanaka (1989a) (Komet Tsirk 1989). However, the energetic cosmic-ray ion processed molecules could provide a kind of "glue" binding the comet dust more strongly than the original ice, thus leading to larger dust particles and reducing the efficiency of dust as a parent molecule source of $\mathrm{C}_{2}$ and $\mathrm{CN}$.

\section{OBSERVATIONAL UNIQUENESS OF COMET YANAKA (1988r)}

So, wherein is Comet Yanaka (1988r) so different from other comets, new or otherwise? We see in Table 1, where some comets are given for comparison, that the largest difference between comet Yanaka (1988r) and the others is in the relative smallness of the aperture used to observe it whether defined as a mean radius or as a maximum length at the comet. The dust-to-gas ratio calculated in a uniform way (Singh et al. 1992) with the others is also smaller. We suggest that in this combination of differences may lie a basis for understanding the uniqueness of the $\mathrm{C}_{2}, \mathrm{CN}$ depletion in Comet Yanaka (1988r).

The basic requirements for the comet dust to evaporate enough organics to provide a significant source of distributed small molecules are that it be hot (hotter than blackbody) and that it be very porous. This was demonstrated quantitatively for porous dust in the Halley dust size spectrum being absolutely required to account for one-half of the $\mathrm{CO}$ being a distributed molecule (Greenberg \& Hage 1990) and should be equally demonstrable for other small molecules. A further enhancement occurs if the comet dust itself fragments as was seen to occur in comet P/Halley (Simpson et al. 1987; Vaisberg et al. 1987). This is because the breakup leads not only to small particles, and a consequent increase in surface-to-mass ratio, but also to generally hotter dust (Greenberg \& Hage 1990). Note that the dust is expected to be hotter further out in the coma because of its size properties, not because it has longer to heat up. Thermal equilibrium always occurs in the order of seconds for all the particles. The increase in surface-to-mass ratio, in addition to enhancing the rate of evaporation, also enhances the scattered continuum radiation. Thus the dust-togas ratio calculated from the scattered flux is higher the farther out one includes the coma. It is therefore to be expected that the dust to gas ratio $(d: g)$ is larger for Yanaka (1989a) than for Yanaka (1988r). If the angular scattering distribution function

TABLE 1

Dust and Dust-to-Gas Ratio in SOME COMETs ${ }^{a}$

\begin{tabular}{|c|c|c|c|c|}
\hline Parameter & $\begin{array}{c}\text { Comet } \\
\text { Yanaka (1988r) }\end{array}$ & $\begin{array}{c}\text { Comet } \\
\text { Yanaka (1989a) }\end{array}$ & $\begin{array}{c}\text { Comet } \\
\text { P/Halley (1982i) }\end{array}$ & $\begin{array}{c}\text { Comet } \\
\text { P/Brorsen-Metcalf } \\
(1989 o)^{\mathrm{b}}\end{array}$ \\
\hline $\begin{array}{l}\text { Projected mean radius }{ }^{\mathrm{c}} \text { and } \\
\quad \text { diaphragm at the comet }(\mathrm{km}) \ldots \ldots \ldots \ldots\end{array}$ & $\begin{array}{c}1870 \\
(660 \times 16,470)\end{array}$ & $\begin{array}{c}9580 \\
(3420 \times 84,200)\end{array}$ & $\begin{array}{c}10,550 \\
(2400 \times 145,600)\end{array}$ & 9310 \\
\hline Heliocentric distance (AU) .............. & 0.932 & 2.11 & 0.87 & 0.91 \\
\hline Geocentric distance (AU) ............... & 0.367 & 1.88 & 1.32 & 0.62 \\
\hline Sun-Comet-Earth angle ................. & $90^{\circ}$ & $28^{\circ}$ & $49^{\circ}$ & $80^{\circ}$ \\
\hline $\begin{array}{l}\text { Scattering function } \mathrm{H}_{2} \mathrm{O} \\
\quad \text { production rate }\left(\mathrm{s}^{-1}\right) \ldots \ldots \ldots \ldots \ldots \ldots\end{array}$ & $\begin{array}{c}0.522 \\
3.74 \times 10^{28}\end{array}$ & $\begin{array}{c}0.580 \\
11.0 \times 10^{28}\end{array}$ & $\begin{array}{c}0.492 \\
65.8 \times 10^{28}\end{array}$ & $\begin{array}{c}0.464 \\
9.2 \times 10^{28}\end{array}$ \\
\hline Nuclear radius $(\mathrm{km}) \ldots . . .$. & 1.4 & 2.3 & 5.6 & 10 \\
\hline 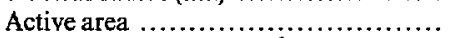 & $10 \%$ & $10 \%$ & $10 \%$ & $10 \%$ \\
\hline $\begin{array}{l}\text { Continuum fiux at } 6250 \AA \\
\quad\left(\text { ergs } \mathrm{cm}^{-2} \mathrm{~s}^{-1} \AA^{-1}\right) \ldots\end{array}$ & $9.85 \times 10^{-15}$ & $1.97 \times 10^{-15}$ & $4.23 \times 10^{-13}$ & $\begin{array}{l}1.26 \times 10^{-13} \\
(\text { at } 484.5 \mathrm{~nm})\end{array}$ \\
\hline Dust mass-loss rate $\left(\mathrm{g} \mathrm{s}^{-1}\right) \ldots \ldots \ldots \ldots$ & $3.04 \times 10^{4}$ & $2.24 \times 10^{5}$ & $3.42 \times 10^{6}$ & $1.22 \times 10^{5}$ \\
\hline Gas loss rate $\left(\mathrm{g} \mathrm{s}^{-1}\right) \ldots \ldots \ldots \ldots \ldots \ldots \ldots$ & $1.23 \times 10^{6}$ & $3.61 \times 10^{6}$ & $2.16 \times 10^{7}$ & $3.00 \times 10^{6}$ \\
\hline Dust-to-gas ratio...$\ldots \ldots \ldots \ldots \ldots \ldots \ldots$ & 0.025 & 0.062 & 0.158 & 0.041 \\
\hline 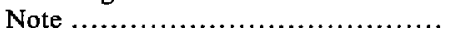 & New & New & Periodic & Periodic \\
\hline
\end{tabular}

a These calculations were done using the following parameters as given in Newburn \& Spinrad 1989: grain albedo =0.04, comet nuclear density $=0.5 \mathrm{~g} \mathrm{~cm}^{-3}$, and particle density $=1 \mathrm{~g} \mathrm{~cm}^{-3}$. Since we are only interested in relative values between the comets, the particular parameters chosen are not critical. As it happens, we would rather have chosen comet nuclear density $=0.3 \mathrm{~g} \mathrm{~cm}^{-3}$ and particle density $<0.1 \mathrm{~g} \mathrm{~cm}^{-3}$ based on Greenberg \& Hage 1990.

b Baratta et al. 1991 .

c $\pi \vec{r}^{2}=(a \times b)$. 
for the comet dust is relatively forward throwing in Yanaka (1988r), then the $d: g$ for Yanaka (1988r) should also be raised accordingly relative to Yanaka (1989a). For the latter the mean phase angle is $90^{\circ}$, whereas for the former it is only $28^{\circ}$ so that the enhancement in $d: g$ for Yanaka (1989a) relative to Yanaka $(1988 \mathrm{r})$ should be approximately $\Phi\left(90^{\circ}\right) / \Phi\left(28^{\circ}\right)$ where $\Phi$ is the mean value of the phase function at the respective angles. This assumes a uniform particle size distribution function over very unequal coma dimensions. The generally used phase function is rather flat between $0^{\circ}$ and $90^{\circ}$ as is true for fluffy particles (Greenberg 1982b), but if the dust in new comets is better glued together, then the dust within the smaller coma of Yanaka (1988r) would be substantially larger than average and be more forward-throwing than that for Yanaka (1989a). A further enhancement in the ratio of light-continuum-based $d: g$ between the two comets should be considered. It is to be remembered that these $d: g$ values are not the actual ones but only those derived from the scattered light fluxes. We now draw the inference that, if the comet dust in Yanaka (1989a) had not been fragmented (relative to Yanaka [1988r]) its light flux would have been substantially lower than observed. With respect to Yanaka (1988r) we infer that because the dust and the lack of $\mathrm{C}_{2}$ and $\mathrm{CN}$ are only observed close to the nucleus, not only is the apparent $d: g$ low, but the dust size distribution is loaded with larger sizes which, because of their relatively low temperatures and reduced total surface area, are inhibited from giving rise to the rapid evaporation of the organic refractory molecules.

As a corollary, in all cases the $d: g$ calculated from the continuum flux cannot approach the actual $d: g$ because the latter is dominated by the larger grains (McDonnell et al. 1987).

\section{DEDUCTIONS FROM OTHER COMETS}

Some of the observed infrared characteristics of the dynamically new comet Austin (1990V) by Hanner et al. (1993) would seem to fit in with the idea that the outer layers of a new comet have been modified so that the dust which comes off in the first solar passage tends to be larger and less fragile than that of periodic comets. Such a comet would appear to be dust poor because the large dust grains are cooler than small ones and furthermore, as predicted in Greenberg \& Hage (1990), should exhibit weaker $10 \mu \mathrm{m}$ emission as, in fact, observed by Hanner et al. (1993). Interestingly, although one might expect the cosmic-ray-produced organic material to be more abundant than normal, the $3.4 \mu \mathrm{m}$ emission is inhibited by the deficiency in small grains, a requirement described by Greenberg \& Hage (1990). It must be reiterated that the strength of the silicate band depends not only on the abundance of silicate and the number of small grains but equally on the organic coating, the submicron size of the units, and the fluffy character of the dust. In the $10 \mu \mathrm{m}$ spectral observation of comet Austin (1990V) the geocentric distance of $0.456 \mathrm{AU}$ and the field of view of 9.4 yield an effective aperture radius at the comet of $1554 \mathrm{~km}$ which is even smaller than that used to observe Yanaka (1988r) so that the same general arguments about the dust apply; that is, one should not expect to see the silicate feature. For the 3.4 $\mu \mathrm{m}$ region the radius was even smaller, being $R \approx 1015 \mathrm{~km}$, further reducing the chance of observing the organic signature. The photometric observations in the 4.7-20 $\mu \mathrm{m}$ range were made with an aperture radius of $\sim 3600 \mathrm{~km}$, and for 1.2-4.7 $\mu \mathrm{m}$ the effective mean radius was $3390 \mathrm{~km}$ (rectangular $4520 \times 7989 \mathrm{~km}^{2}$ ) so that all the observations reported by
Hanner et al. for comet Austin (1990V) were made with apertures similar to that for Yanaka (1988r). Thus the fact that it appears to be dust poor is entirely to be expected because the dust size distribution should be weighted toward larger grains as also inferred by Hanner et al. (1993). When a much larger field of view $\left(560 \times 45,000 \mathrm{~km}^{2}=\right.$ slit dimension $\left.2.5 \times 200^{\prime \prime}\right)$ was used for comet Austin (1990V) ( $\left.\equiv 1989 c_{1}\right)$, there was enough fragmentation "far out" to permit observations of $\mathrm{C}_{2}$ and CN (Tegler et al. 1992).

Although many of the observations of comet IRAS-ArakiAlcock were made with small apertures, this comet is not a dynamically new one so one of the major criteria determining the dust properties close to the nucleus, namely the presence of a crustal layer processed over 4.5 Gyr to a depth of "some meters" (Strazzulla \& Johnson 1991), does not apply. Nevertheless, even with fields of view as small as $1680 \times 10,000$ to $6770 \times 11,200 \mathrm{~km}^{2}$ it was suggested that changes in the infrared spectra of the coma and tail could be attributed to the particle mean size decreasing with increasing distance from the nucleus. Thus the fact that the $10 \mu \mathrm{m}$ silicate emission was not observed by Feierberg et al. (1984) could possibly be attributed to the deficiency, close to the nucleus, of enough small (or fluffy small) particles as required by Greenberg \& Hage (1990) to provide the excess spectral emission. Insofar as the $\mathrm{C}_{2}$ emission is concerned Lutz \& Wagner (1986) noted that "increases in $\mathrm{C}_{2}$ emission and continuum flux are essentially indistinguishable" which could imply that at least a significant fraction of the parent molecules of $\mathrm{C}_{2}$ are directly associated with the dust, for example, via evaporation. This interpretation is similar to that of Festou et al. (1987) where it is concluded that $\mathrm{C}_{2}$ radicals have at least two parents, one of which could be the same as that of the $\mathrm{C}_{3}$ radical. Possible candidates for $\mathrm{CN}$ sources suggested by Bockelee-Morvan et al. (1984) are $\mathrm{C}_{2} \mathrm{~N}_{2}$, $\mathrm{CH}_{3} \mathrm{CN}, \mathrm{HC}_{3} \mathrm{~N}, \mathrm{HCN}$, but one could also suggest the source being the evaporating organics from the interstellar grain mantles (Briggs et al. 1992). The source of $\mathrm{CN}$ in comet $\mathrm{P} / \mathrm{Halley}$ has been often so attributed because of its spatial distribution in the coma (A'Hearn et al. 1986a, b). The distribution of $\mathrm{C}_{2}$ as described in Oliversen, Hollis, \& Brown (1985) and the possible sources of $\mathrm{CN}, \mathrm{C}_{2}$ described above are consistent with a substantial fraction of the parent molecule being evaporated off the organic material in the dust (Briggs et al. 1992). While IRAS-Araki-Alcock and Halley are not new comets their general dust properties (fluffiness, etc.) may, nevertheless, be indicative of the dust in new comets if proper account is taken of the anticipated differences described above. We note that microwave observations of comet IRAS-ArakiAlcock (1983VII) were interpreted by Sekanina (1988) to imply that the "comet's interior was extremely cold" which is consistent with the constraint on the internal heating from the Sun through a very fluffy structure (Greenberg, Grim, \& van Ijzendoorn 1986).

\section{CONCLUSIONS}

In summary, we propose that the relative depletion of the minor species $\mathrm{C}_{2}$ and $\mathrm{CN}$ in comet Yanaka (1988r) comes about because (1) the parent source of these molecules is relatively tightly bound within the comet dust of a new comet whose crust has been processed by cosmic rays while in the Oort cloud, (2) the cosmic-ray-processed dust is less fragile (is better glued together) than that of a periodic comet so that its size distribution is weighted toward larger cooler particles than 
those of an old comet, and (3) the small central observation

$\therefore$ diaphragm further exaggerates the size distribution effect by

i not allowing time for fragmentation to occur until farther out

In in the coma. The low surface-to-mass ratio and the relative II coolness of the dust within the region of observation of Yanaka

- (1988r) combine in a nonlinear way to inhibit severely the "usual" sources of $\mathrm{C}_{2}$ and $\mathrm{CN}$. This suggests the importance of observing comets with small and progressively larger diaphragms, or small diaphragms at different parts of the coma, to obtain new information about dust fragmentation and its contribution to molecular species in the coma.

This work was supported by CNPq, Brasília under contract 451443/92-6 (NV) and by NASA grant NGR 33-018-148. J. M. G. thanks the CNPq for making possible a stay at the Institute of Astronomy and Geophysics-USP. We thank W. Huebner for some helpful suggestions.
A'Hearn, M. F., Hoban, S., Birch, P. V., Bowers, C., Martin, R., \& Klinglesmith, D. A. 1986a, Nature, 324, 649 1986 , ESA SP-250, vol. 1,483

A'Hearn, M. F., Schleicher, D. G., Feldman, P. D., Millis, R. L., \& Thompson, D. T. 1984, AJ, 89, 579

Baratta, G. A., Catalano, F. A., Leone, F., \& Strazzulla, G. 1991, A\&A, 251, 349

Bockelee-Moryan, D., Crovisier, J., Baudry, A., Despois, D., Perault, M. Irvine, W. M., Schloerb, F. P., \& Swade, D. 1984, A\&A, 141, 411

Briggs, R., Ertem, G., Ferris, J. P., Greenberg, J. M., McCain, P. J., Mendoza Gomaz, C. X., \& Schutte, W. 1992. Origins of Life and Evolution of the Biosphere, 22, 287

Brooke, T. Y., Knacke, R. F., Owen, T. C., \& Tokunaga, A. T. 1989, ApJ, 336, 971

Chlewicki, G. 1986, Ph.D. thesis, Univ. Leiden

Clairemidi, J., Rouffelot, P., \& Moreels, G. 1991, in Origin and Evolution of Interplanetary Dust, ed. A. C. Levasseur-Regourd \& H. Hasegawa (Dordrecht: Kluwer), 217

Delsemme, A. H. 1982, in Comets, ed. L. L. Wilkening (Tucson: Univ. Arizona Press), 85

d'Hendecourt, L. B., Allamandola, L. J., \& Greenberg, J. M. 1985, A\&A, 152, 130

Eberhardt, P., et al. 1987, A\&A, 187, 481

Feierberg, M. A., Witteborn, F. C., Johnson, J. R., \& Campkins, H. 1984 Icarus, 60,449

Festou, M. C., Ecrenaz, T., Boisson, C., Pedersen, H., \& Tarenghi, M. 1987, A\&A, 174, 299

Fink, U. 1992, Science, 257, 1926

Geiss, J. 1987, A\&A, 187, 859

Greenberg, J. M. 1982a, in Submillimetre Wave Astronomy, ed. J. E. Beckman \& J. P. Phillips (Cambridge: Cambridge Univ. Press), 261 131

. 1982b, in Comets, ed. L. L. Wilkening (Tucson: Univ. Arizona Press),

. 1983, in Cometary Exploration, ed. T. I. Gombosi (Budapest: Hungarian Academy of Sciences), 23

1991, in Cosmic Rays, Supernovae and the Interstellar Medium, ed. M. M. Shapiro \& J. P. Weifel (Dordrecht: Kluwer), 57

Greenberg, J. M., Grim, R., \& van Ijzendoorn, L. J. 1986, in Asteroids, Comets, Meteors II, ed. C.-I. Lagerkvist et al. (Uppsala: Reprocentrum HSC), 218
Greenberg, J. M., \& Hage, J. I. 1990, ApJ, 361, 260

Hanner, M. S., Russell, R. W., Lynch, D. K., \& Brooke, T. Y. 1993, Icarus, 101, 64

Jessberger, E. K., Kissel, J., \& Rahe, J. 1989, in Origin and Evolution of Planetary and Satellite Atmospheres, ed. S. K. Atreya, J. B. Pollack, \& M. S. Matthews (Tucson: Univ. Arizona Press), 167

Kissel, J., \& Krueger, F. R. 1987, Nature, 326, 755

Komet Tsirk. 1988, No. 400, 7

. 1989 , No. 401,1

Larson, H. P., Weaver, H. A., Mumma, M. J., \& Drapatz, S. 1989, ApJ, 338, 1106

Lutz, B. L., \& Wagner, R. M. 1986, ApJ, 308, 993

Mathis, J. S., Rumpl, W. \& Nordsieck, K. H. 1977, ApJ, 217, 425

McDonnell, J. A. M., et al. 1987, A\&A, 187, 719

Mumma, M., Weissman, P., \& Stern, A. 1993, in Protostars and Planets III, ed. E. H. Levy, J. I. Lunine, \& M. S. Matthews (Tucson: Univ. Arizona Press), 1177

Newburn, R. L., \& Spinrad, H. 1989, AJ, 97, 552

Oliversen, R. J., Hollis, J. M., \& Brown, L. W. 1985, Icarus, 63, 339

Pollack, J. B., \& Bodenheimer, P. 1989, in Origin and Evolution of Planetary and Satellite Atmospheres, ed. S. K. Atreya, J. B. Pollack, \& M. S. Matthews (Tucson: Univ. Arizona Press), 564

Sekanina, Z. 1988, AJ, 95, 1876

Simpson, J. A., Rabinowitz, D., Tuzzolino, A. J., Ksanfomality, L. V., \& Sagdeev, R. Z. 1987, A\&A, 187, 742

Singh, P.D., de Almeida, A. A., \& Huebner, W. F. 1992, AJ, 104, 848

Singh, P. D., Huebner, W. F., Costa, R. D. D., Landaberry, S. J. C., \& de Freitas Pacheco, J. A. 1993, in preparation

Strazzulla, G., \& Johnson, R. E. 1991, in Comets in the Post-Halley Era, ed. R. L. Newburn et al. (Dordrecht: Kluwer), vol. 1, 243

Tegler, S. C., Campins, H., Larson, S., Kleine, M., Kelly, D., \& Rieke, M. 1992, ApJ, 396, 711

Vaisberg, O. L., Smirnov, V., Omelchenko, A., Gorn, L., \& Tovlev, M. 1987, A\&A, 187,753

van Dishoeck, E. F., Blake, G. A., Draine, B. T., \& Lunine, J. I. 1993, in Protostars and Planets III, ed. E. H. Levy, J. I. Lunine, \& M. S. Matthews (Tucson: Univ. Arizona Press), 163

Walker, R. G., Aumann, H. H., Davies, J., Green, S., de Jong, T., Houck, J. R., \& Soifer, B. T. 1984, ApJ, 278, L11 